Acta Crystallographica Section E

\section{Structure Reports}

Online

ISSN 1600-5368

\section{Redetermination of $\alpha$-D-glucosamine hydrochloride: elucidation of the hydrogen-bonding scheme}

\author{
William T. A. Harrison, ${ }^{a *}$ H. S. Yathirajan, ${ }^{b}$ B. Narayana, \\ T. V. Sreevidya ${ }^{c}$ and B. K. Sarojini ${ }^{d}$ \\ a Department of Chemistry, University of Aberdeen, Meston Walk, Aberdeen AB24 \\ 3UE, UK, ${ }^{\mathbf{b}}$ Department of Studies in Chemistry, University of Mysore, Manasagan- \\ gotri, Mysore 570 006, India, ${ }^{\mathbf{c}}$ Department of Chemistry, Mangalore University, \\ Mangalagangotri 574 199, India, and dDepartment of Chemistry, P. A. College of \\ Engineering, Mangalore 574 153, India \\ Correspondence e-mail: w.harrison@abdn.ac.uk
}

Received 17 May 2007; accepted 18 May 2007

Key indicators: single-crystal X-ray study; $T=295 \mathrm{~K}$; mean $\sigma(\mathrm{C}-\mathrm{C})=0.002 \AA$; $R$ factor $=0.029 ; w R$ factor $=0.075 ;$ data-to-parameter ratio $=18.5$.

The previously reported crystal structures [Chu \& Jeffery (1965). Proc. R. Soc. London Ser. A, 285, 470-479; Chandrasekharan \& Mallikarjunan (1969). Z. Kristallogr. 129, 29] of the title compound, $\mathrm{C}_{6} \mathrm{H}_{14} \mathrm{NO}_{5}{ }^{+} \cdot \mathrm{Cl}^{-}$, have been confirmed to higher precision, and the $\mathrm{H}$ atoms located, allowing the elucidaton of the hydrogen-bonding network. A combination of $\mathrm{O}-\mathrm{H} \cdots \mathrm{O}, \mathrm{N}-\mathrm{H} \cdots \mathrm{O}, \mathrm{O}-\mathrm{H} \cdots \mathrm{Cl}$ and $\mathrm{N}-\mathrm{H} \cdots \mathrm{Cl}$ links results in a three-dimensional network. Considered by themselves, the inter-cation $\mathrm{O}-\mathrm{H} \cdots \mathrm{O}$ and $\mathrm{N}-\mathrm{H} \cdots \mathrm{O}$ hydrogen bonds result in undulating (001) layers. The configurations of the chiral $\mathrm{C}$ atoms are: $\mathrm{C} 1 S, \mathrm{C} 2 R, \mathrm{C} 3 R, \mathrm{C} 4 S$ and $\mathrm{C} 5 R$.

\section{Related literature}

For an earlier structure determination of the title compound, in which the $\mathrm{H}$ atoms were not located, and background literature, see: Chu \& Jeffery (1965); Chandrasekharan \& Mallikarjunan (1969); Allen et al. (1987).

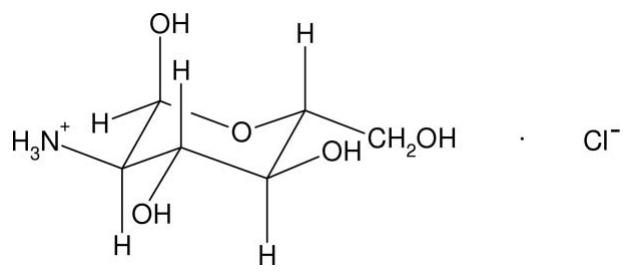

\section{Experimental}

\section{Crystal data}

$\mathrm{C}_{6} \mathrm{H}_{14} \mathrm{NO}_{5}^{+} \cdot \mathrm{Cl}^{-}$

$M_{r}=215.63$

$$
\begin{aligned}
& b=9.2140(6) \AA \\
& c=7.7650(5) \AA \\
& \beta=112.884(1) \circ \\
& V=471.12(5) \AA^{3} \\
& Z=2
\end{aligned}
$$

Data collection

Bruker SMART 1000 CCD diffractometer

Absorption correction: multi-scan (SADABS; Bruker, 1999)

$T_{\min }=0.851, T_{\max }=0.944$

Refinement

$R\left[F^{2}>2 \sigma\left(F^{2}\right)\right]=0.029$

$w R\left(F^{2}\right)=0.075$

$S=1.07$

2217 reflections

120 parameters

1 restraint
Mo $K \alpha$ radiation $T=295$ (2) K $0.42 \times 0.30 \times 0.15 \mathrm{~mm}$

3941 measured reflections 2217 independent reflections 2124 reflections with $I>2 \sigma(I)$ $R_{\text {int }}=0.020$ $\mu=0.40 \mathrm{~mm}^{-1}$

\section{Table 1}

Hydrogen-bond geometry $\left(\AA{ }^{\circ}\right)$.

\begin{tabular}{lllll}
\hline$D-\mathrm{H} \cdots A$ & $D-\mathrm{H}$ & $\mathrm{H} \cdots A$ & $D \cdots A$ & $D-\mathrm{H} \cdots A$ \\
\hline $\mathrm{N} 1-\mathrm{H} 1 B \cdots \mathrm{O} 5^{\text {i }}$ & 0.89 & 1.89 & $2.7772(17)$ & 172 \\
$\mathrm{~N} 1-\mathrm{H} 1 C \cdots 4^{\text {ii }}$ & 0.89 & 2.15 & $2.8930(19)$ & 141 \\
$\mathrm{~N} 1-\mathrm{H} 1 D \cdots \mathrm{Cl} 1^{\mathrm{iii}}$ & 0.89 & 2.38 & $3.1744(13)$ & 149 \\
$\mathrm{O} 2-\mathrm{H} 2 \cdots \mathrm{Cl} 1^{\text {iv }}$ & 0.82 & 2.35 & $3.1448(12)$ & 162 \\
$\mathrm{O} 3-\mathrm{H} 3 \cdots \mathrm{Cl} 1^{\mathrm{v}}$ & 0.84 & 2.35 & $3.1911(14)$ & 173 \\
$\mathrm{O} 4-\mathrm{H} 4 \cdots \mathrm{Cl} 1$ & 0.82 & 2.35 & $3.1667(14)$ & 175 \\
$\mathrm{O}^{\mathrm{V}}-\mathrm{H} 5 \cdots \mathrm{O}^{\mathrm{v}}$ & 0.81 & 1.95 & $2.7373(17)$ & 163 \\
\hline
\end{tabular}

Symmetry codes: (i) $-x, y+\frac{1}{2},-z+2$; (ii) $-x+1, y+\frac{1}{2},-z+2$; (iii) $x, y, z+1$; (iv) $x-1, y, z ;(\mathrm{v})-x+1, y-\frac{1}{2},-z+2$.

Data collection: SMART (Bruker, 1999); cell refinement: SAINT (Bruker, 1999); data reduction: $S A I N T$; $\operatorname{program}(\mathrm{s})$ used to solve structure: SHELXS97 (Sheldrick, 1997); program(s) used to refine structure: SHELXL97 (Sheldrick, 1997); molecular graphics: ORTEP-3 (Farrugia, 1997); software used to prepare material for publication: SHELXL97.

We thank Strides Arco Labs, Mangalore, India for the gift sample of glucosamine hydrochloride. BKS thanks AICTE, Government of India, New Delhi for financial assistance under the Career Award for Young Teachers scheme.

Supplementary data and figures for this paper are available from the IUCr electronic archives (Reference: BT2375).

\section{References}

Allen, F. H., Kennard, O., Watson, D. G., Brammer, L., Orpen, A. G. \& Taylor, R. (1987). J. Chem. Soc. Perkin Trans. 2, pp. S1-19.

Bruker (1999). SMART, SAINT and SADABS. Bruker AXS Inc., Madison, Wisconsin, USA.

Chandrasekharan, R. \& Mallikarjunan, M. (1969). Z. Kristallogr. 129, 29.

Chu, S. S. C. \& Jeffery, G. A. (1965). Proc. R. Soc. London Ser. A, 285, 470479.

Farrugia, L. J. (1997). J. Appl. Cryst. 30, 565.

Flack, H. D. (1983). Acta Cryst. A39, 876-881.

Sheldrick, G. M. (1997). SHELXS97 and SHELXL97. University of Göttingen, Germany. 


\section{supplementary materials}




\title{
Redetermination of $\alpha$-D-glucosamine hydrochloride: elucidation of the hydrogen-bonding scheme
}

\author{
W. T. A. Harrison, H. S. Yathirajan, B. Narayana, T. V. Sreevidya and B. K. Sarojini
}

\section{Comment}

The present study confirms the previous structure determinations (Chu \& Jeffery, 1965; Chandrasekharan \& Mallikarjunan, 1969) of the title compound, (I), to modern standards of precision. All the geometrical values for (I) (Fig. 1) fall within their expected ranges (Allen et al., 1995) and the six-membered ring is well described as a chair: for atoms C1, C2, C4 and C5, the r.m.s. deviation from their mean plane is $0.021 \AA$ : $\mathrm{C} 3$ and $\mathrm{O} 1$ deviate from the plane by -0.652 (2) $\AA$ and 0.6477 (19) Å, respectively.

Here, the $\mathrm{H}$ atoms have been located, allowing the hydrogen-bonding scheme, involving a combination of $\mathrm{O}-\mathrm{H} \cdots \mathrm{O}$, $\mathrm{N}-\mathrm{H} \cdots \mathrm{O}, \mathrm{O}-\mathrm{H} \cdots \mathrm{Cl}$ and $\mathrm{N}-\mathrm{H} \cdots \mathrm{Cl}$ links, to be elucidated (Table 1). The inter-cation $\mathrm{N}-\mathrm{H} \cdots \mathrm{O}$ and $\mathrm{O}-\mathrm{H} \cdots \mathrm{O}$ bonds result in undulating (001) sheets (Fig. 2).

\section{Experimental}

A sample of glucosamine hydrochloride was obtained from Strides Arco labs, Mangalore, India and recrystallized from water to yield colourless chunks of (I). m.p.: 449-451 K.

\section{Refinement}

The O-bound hydrogen atoms were located in a difference map and refined as riding in their as-found relative positions with $U_{\text {iso }}(\mathrm{H})=1.2 U_{\text {eq }}(\mathrm{O})$. The other hydrogen atoms were geometrically placed $(\mathrm{C}-\mathrm{H}=0.97-0.98 \AA, \mathrm{N}-\mathrm{H}=0.89 \AA)$ and refined as riding with $U_{\text {iso }}(\mathrm{H})=1.2 U_{\text {eq }}$ (carrier). The $-\mathrm{NH}_{3}{ }^{+}$group was allowed to rotate, but not to tip, to best fit the electron density.

\section{Figures}

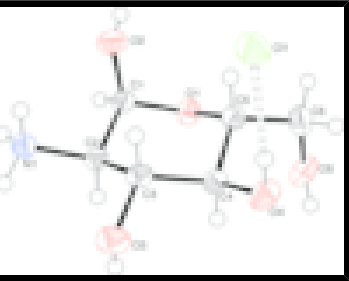

Fig. 1. View of the molecular structure of (I) showing 50\% displacement ellipsoids (arbitrary spheres for the $\mathrm{H}$ atoms). The hydrogen bond is shown as a double dashed line. 


\section{supplementary materials}

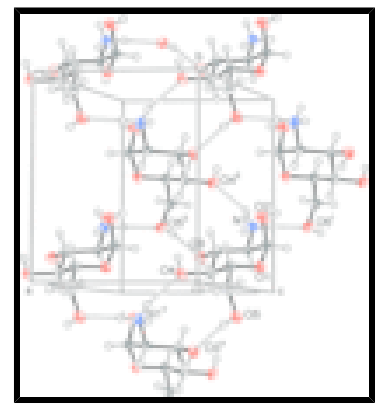

Fig. 2. Part of an (001) sheet of cations in (I) linked by $\mathrm{O}-\mathrm{H} \cdots \mathrm{O}$ and $\mathrm{N}-\mathrm{H} \cdots \mathrm{O}$ hydrogen bonds. Symmetry codes as in Table 1.

\section{$\alpha$-D-glucosamine hydrochloride}

\section{Crystal data}

$\mathrm{C}_{6} \mathrm{H}_{14} \mathrm{NO}_{5}^{+} \cdot \mathrm{Cl}^{-}$

$F_{000}=228$

$M_{r}=215.63$

$D_{\mathrm{x}}=1.520 \mathrm{Mg} \mathrm{m}^{-3}$

Monoclinic, $P 2_{1}$

Mo $K \alpha$ radiation

$\lambda=0.71073 \AA$

Hall symbol: $\mathrm{P} 2 \mathrm{yb}$

Cell parameters from 2993 reflections

$a=7.1474(5) \AA$

$\theta=5.0-30.0^{\circ}$

$b=9.2140(6) \AA$

$\mu=0.40 \mathrm{~mm}^{-1}$

$c=7.7650(5) \AA$

$T=295(2) \mathrm{K}$

$\beta=112.884(1)^{\circ}$

Chunk, colourless

$V=471.12(5) \AA^{3}$

$0.42 \times 0.30 \times 0.15 \mathrm{~mm}$

$Z=2$

\section{Data collection}

Bruker SMART1000 CCD

diffractometer

2217 independent reflections

Radiation source: fine-focus sealed tube

2124 reflections with $I>2 \sigma(I)$

Monochromator: graphite

$R_{\text {int }}=0.020$

$T=295(2) \mathrm{K}$

$\theta_{\max }=30.0^{\circ}$

$\omega$ scans

$\theta_{\min }=5.0^{\circ}$

Absorption correction: multi-scan

SADABS (Bruker, 1999)

$h=-10 \rightarrow 10$

$T_{\min }=0.851, T_{\max }=0.944$

$k=-9 \rightarrow 12$

3941 measured reflections

$l=-10 \rightarrow 9$

\section{Refinement}

Refinement on $F^{2}$

Least-squares matrix: full

$R\left[F^{2}>2 \sigma\left(F^{2}\right)\right]=0.029$

$w R\left(F^{2}\right)=0.075$

Hydrogen site location: difmap $(\mathrm{O}-\mathrm{H})$ and geom (others)

$\mathrm{H}$-atom parameters constrained

$$
w=1 /\left[\sigma^{2}\left(F_{\mathrm{o}}{ }^{2}\right)+(0.0452 P)^{2}+0.0391 P\right]
$$

where $P=\left(F_{\mathrm{o}}^{2}+2 F_{\mathrm{c}}^{2}\right) / 3$

$(\Delta / \sigma)_{\max }<0.001$ 
$S=1.07$

2217 reflections

120 parameters

1 restraint

Primary atom site location: structure-invariant direct methods

Secondary atom site location: difference Fourier map Flack parameter: 0.07 (5)

$\Delta \rho_{\min }=-0.21$ e $\AA^{-3}$
$\Delta \rho_{\max }=0.27$ e $\AA^{-3}$

Extinction correction: SHELXL,

$\mathrm{Fc}^{*}=\mathrm{kFc}\left[1+0.001 \times \mathrm{Fc}^{2} \lambda^{3} / \sin (2 \theta)\right]^{-1 / 4}$

Extinction coefficient: 0.042 (7)

Absolute structure: Flack (1983), 761 Friedel pairs

\section{Special details}

Geometry. All e.s.d.'s (except the e.s.d. in the dihedral angle between two l.s. planes) are estimated using the full covariance matrix. The cell e.s.d.'s are taken into account individually in the estimation of e.s.d.'s in distances, angles and torsion angles; correlations between e.s.d.'s in cell parameters are only used when they are defined by crystal symmetry. An approximate (isotropic) treatment of cell e.s.d.'s is used for estimating e.s.d.'s involving l.s. planes.

Refinement. Refinement of $\mathrm{F}^{2}$ against ALL reflections. The weighted $R$-factor $w R$ and goodness of fit $\mathrm{S}$ are based on $\mathrm{F}^{2}$, conventional $R$-factors $R$ are based on $\mathrm{F}$, with $\mathrm{F}$ set to zero for negative $\mathrm{F}^{2}$. The threshold expression of $\mathrm{F}^{2}>2 \operatorname{sigma}\left(\mathrm{F}^{2}\right)$ is used only for calculating $R$-factors(gt) etc. and is not relevant to the choice of reflections for refinement. $R$-factors based on $\mathrm{F}^{2}$ are statistically about twice as large as those based on F, and R- factors based on ALL data will be even larger.

Fractional atomic coordinates and isotropic or equivalent isotropic displacement parameters $\left(\AA^{2}\right)$

$\begin{array}{lllll} & x & y & z & U_{\text {iso }} * U_{\text {eq }} \\ \text { C1 } & 0.0118(2) & 0.23568(18) & 0.9307(2) & 0.0254(3) \\ \text { H1A } & -0.0990 & 0.2269 & 0.9748 & 0.030^{*} \\ \text { C2 } & 0.2141(2) & 0.22407(17) & 1.09837(19) & 0.0224(3) \\ \text { H2A } & 0.2115 & 0.1363 & 1.1687 & 0.027^{*} \\ \text { C3 } & 0.3952(2) & 0.21459(18) & 1.0412(2) & 0.0234(3) \\ \text { H3A } & 0.4058 & 0.3070 & 0.9830 & 0.028^{*} \\ \text { C4 } & 0.3592(2) & 0.09447(18) & 0.89614(19) & 0.0233(3) \\ \text { H4A } & 0.3528 & 0.0017 & 0.9553 & 0.028^{*} \\ \text { C5 } & 0.1553(2) & 0.11884(19) & 0.7337(2) & 0.0248(3) \\ \text { H5A } & 0.1577 & 0.2125 & 0.6748 & 0.030^{*} \\ \text { C6 } & 0.1010(3) & 0.0004(2) & 0.5870(2) & 0.0332(4) \\ \text { H6A } & -0.0254 & 0.0255 & 0.4850 & 0.040^{*} \\ \text { H6B } & 0.2059 & -0.0051 & 0.5371 & 0.040^{*} \\ \text { N1 } & 0.23946(19) & 0.35206(15) & 1.22281(17) & 0.0266(3) \\ \text { H1B } & 0.1307 & 0.3608 & 1.2511 & 0.032 * \\ \text { H1C } & 0.2535 & 0.4319 & 1.1644 & 0.032^{*} \\ \text { H1D } & 0.3494 & 0.3400 & 1.3272 & 0.032^{*} \\ \text { O1 } & -0.00356(15) & 0.12014(13) & 0.80456(15) & 0.0261(2) \\ \text { O2 } & 0.00134(17) & 0.37174(15) & 0.85023(15) & 0.0348(3) \\ \text { H2 } & -0.1164 & 0.3899 & 0.7813 & 0.042^{*} \\ \text { O3 } & 0.57819(17) & 0.19429(15) & 1.20313(17) & 0.0332(3) \\ \text { H3 } & 0.5477 & 0.1193 & 1.2483 & 0.040^{*} \\ \text { O4 } & 0.52073(18) & 0.08680(15) & 0.83229(17) & 0.0325(3) \\ \text { H4 } & 0.1640 & 0.7788 & 0.039^{*}\end{array}$


supplementary materials

$\begin{array}{lllll}\text { O5 } & 0.07990(17) & -0.13730(14) & 0.65823(17) & 0.0354(3) \\ \text { H5 } & 0.1895 & -0.1737 & 0.7165 & 0.042 * \\ \text { C11 } & 0.52602(6) & 0.39359(5) & 0.65041(5) & 0.03359(11)\end{array}$

Atomic displacement parameters $\left(\AA^{2}\right)$

$\begin{array}{lllllll} & U^{11} & U^{22} & U^{33} & U^{12} & U^{13} & U^{23} \\ \mathrm{C} 1 & 0.0204(6) & 0.0265(8) & 0.0268(6) & 0.0012(5) & 0.0064(5) & -0.0039(6) \\ \mathrm{C} 2 & 0.0207(6) & 0.0209(7) & 0.0242(6) & 0.0001(5) & 0.0074(5) & 0.0000(5) \\ \mathrm{C} 3 & 0.0193(6) & 0.0222(8) & 0.0269(6) & -0.0004(5) & 0.0068(5) & -0.0002(5) \\ \text { C4 } & 0.0230(6) & 0.0210(8) & 0.0271(6) & 0.0000(5) & 0.0111(5) & 0.0003(5) \\ \text { C5 } & 0.0255(6) & 0.0244(8) & 0.0252(6) & -0.0004(5) & 0.0106(5) & 0.0007(6) \\ \text { C6 } & 0.0328(8) & 0.0396(10) & 0.0280(7) & -0.0020(7) & 0.0127(6) & -0.0073(7) \\ \text { N1 } & 0.0261(6) & 0.0271(7) & 0.0252(5) & 0.0003(5) & 0.0085(4) & -0.0036(5) \\ \text { O1 } & 0.0217(5) & 0.0266(6) & 0.0293(5) & -0.0030(4) & 0.0092(4) & -0.0067(4) \\ \text { O2 } & 0.0322(5) & 0.0282(8) & 0.0344(5) & 0.0054(5) & 0.0025(4) & 0.0018(5) \\ \text { O3 } & 0.0213(5) & 0.0321(7) & 0.0366(6) & -0.0031(5) & 0.0009(4) & -0.0014(5) \\ \text { O4 } & 0.0308(5) & 0.0306(7) & 0.0429(7) & 0.0047(5) & 0.0217(5) & 0.0018(5) \\ \text { O5 } & 0.0264(5) & 0.0293(8) & 0.0496(7) & -0.0001(5) & 0.0137(5) & -0.0106(5) \\ \text { C11 } & 0.03269(18) & 0.0357(2) & 0.02785(16) & -0.00225(17) & 0.00684(12) & -0.00076(17)\end{array}$

Geometric parameters $\left(\AA,{ }^{\circ}\right)$

$\begin{array}{ll}\mathrm{C} 1-\mathrm{O} 2 & 1.390(2) \\ \mathrm{C} 1-\mathrm{O} 1 & 1.4219(19) \\ \mathrm{C} 1-\mathrm{C} 2 & 1.5273(18) \\ \mathrm{C} 1-\mathrm{H} 1 \mathrm{~A} & 0.9800 \\ \mathrm{C} 2-\mathrm{N} 1 & 1.4902(19) \\ \mathrm{C} 2-\mathrm{C} 3 & 1.5260(19) \\ \mathrm{C} 2-\mathrm{H} 2 \mathrm{~A} & 0.9800 \\ \mathrm{C} 3-\mathrm{O} 3 & 1.4311(17) \\ \mathrm{C} 3-\mathrm{C} 4 & 1.528(2) \\ \mathrm{C} 3-\mathrm{H} 3 \mathrm{~A} & 0.9800 \\ \mathrm{C} 4-\mathrm{O} 4 & 1.4243(16) \\ \mathrm{C} 4-\mathrm{C} 5 & 1.5286(19) \\ \mathrm{C} 4-\mathrm{H} 4 \mathrm{~A} & 0.9800 \\ \mathrm{O} 2-\mathrm{C} 1-\mathrm{O} 1 & 112.90(12) \\ \mathrm{O} 2-\mathrm{C} 1-\mathrm{C} 2 & 108.30(13) \\ \mathrm{O} 1-\mathrm{C} 1-\mathrm{C} 2 & 109.18(12) \\ \mathrm{O} 2-\mathrm{C} 1-\mathrm{H} 1 \mathrm{~A} & 108.8 \\ \mathrm{O} 1-\mathrm{C} 1-\mathrm{H} 1 \mathrm{~A} & 108.8 \\ \mathrm{C} 2-\mathrm{C} 1-\mathrm{H} 1 \mathrm{~A} & 108.8 \\ \mathrm{~N} 1-\mathrm{C} 2-\mathrm{C} 3 & 109.32(12) \\ \mathrm{N} 1-\mathrm{C} 2-\mathrm{C} 1 & 109.48(12) \\ \mathrm{C} 3-\mathrm{C} 2-\mathrm{C} 1 & 112.65(11) \\ \mathrm{N} 1-\mathrm{C} 2-\mathrm{H} 2 \mathrm{~A} & 108.4 \\ \mathrm{C} 3-\mathrm{C} 2-\mathrm{H} 2 \mathrm{~A} & 108.4 \\ \mathrm{C} 1-\mathrm{C} 2-\mathrm{H} 2 \mathrm{~A} & 108.4\end{array}$

$\begin{array}{ll}\mathrm{C} 5-\mathrm{O} 1 & 1.4413(17) \\ \mathrm{C} 5-\mathrm{C} 6 & 1.515(2) \\ \mathrm{C} 5-\mathrm{H} 5 \mathrm{~A} & 0.9800 \\ \mathrm{C} 6-\mathrm{O} 5 & 1.415(2) \\ \mathrm{C} 6-\mathrm{H} 6 \mathrm{~A} & 0.9700 \\ \mathrm{C} 6-\mathrm{H} 6 \mathrm{~B} & 0.9700 \\ \mathrm{~N} 1-\mathrm{H} 1 \mathrm{~B} & 0.8900 \\ \mathrm{~N} 1-\mathrm{H} 1 \mathrm{C} & 0.8900 \\ \mathrm{~N} 1-\mathrm{H} 1 \mathrm{D} & 0.8900 \\ \mathrm{O} 2-\mathrm{H} 2 & 0.8199 \\ \mathrm{O} 3-\mathrm{H} 3 & 0.8407 \\ \mathrm{O} 4-\mathrm{H} 4 & 0.8213 \\ \mathrm{O} 5-\mathrm{H} 5 & 0.8094 \\ \mathrm{O} 1-\mathrm{C} 5-\mathrm{C} 6 & 106.48(12) \\ \mathrm{O} 1-\mathrm{C} 5-\mathrm{C} 4 & 109.03(11) \\ \mathrm{C} 6-\mathrm{C} 5-\mathrm{C} 4 & 113.42(14) \\ \mathrm{O} 1-\mathrm{C} 5-\mathrm{H} 5 \mathrm{~A} & 109.3 \\ \mathrm{C} 6-\mathrm{C} 5-\mathrm{H} 5 \mathrm{~A} & 109.3 \\ \mathrm{C} 4-\mathrm{C} 5-\mathrm{H} 5 \mathrm{~A} & 109.3 \\ \mathrm{O} 5-\mathrm{C} 6-\mathrm{C} 5 & 112.77(12) \\ \mathrm{O} 5-\mathrm{C} 6-\mathrm{H} 6 \mathrm{~A} & 109.0 \\ \mathrm{C} 5-\mathrm{C} 6-\mathrm{H} 6 \mathrm{~A} & 109.0 \\ \mathrm{O} 5-\mathrm{C} 6-\mathrm{H} 6 \mathrm{~B} & 109.0 \\ \mathrm{C} 5-\mathrm{C} 6-\mathrm{H} 6 \mathrm{~B} & 109.0 \\ \mathrm{H} 6 \mathrm{~A}-\mathrm{C} 6-\mathrm{H} 6 \mathrm{~B} & 107.8 \\ & \end{array}$




\begin{tabular}{|c|c|c|c|}
\hline $\mathrm{O} 3-\mathrm{C} 3-\mathrm{C} 2$ & $109.91(12)$ & $\mathrm{C} 2-\mathrm{N} 1-\mathrm{H} 1 \mathrm{~B}$ & 109.5 \\
\hline $\mathrm{O} 3-\mathrm{C} 3-\mathrm{C} 4$ & $112.80(13)$ & $\mathrm{C} 2-\mathrm{N} 1-\mathrm{H} 1 \mathrm{C}$ & 109.5 \\
\hline $\mathrm{C} 2-\mathrm{C} 3-\mathrm{C} 4$ & $109.71(12)$ & $\mathrm{H} 1 \mathrm{~B}-\mathrm{N} 1-\mathrm{H} 1 \mathrm{C}$ & 109.5 \\
\hline $\mathrm{O} 3-\mathrm{C} 3-\mathrm{H} 3 \mathrm{~A}$ & 108.1 & $\mathrm{C} 2-\mathrm{N} 1-\mathrm{H} 1 \mathrm{D}$ & 109.5 \\
\hline $\mathrm{C} 2-\mathrm{C} 3-\mathrm{H} 3 \mathrm{~A}$ & 108.1 & $\mathrm{H} 1 \mathrm{~B}-\mathrm{N} 1-\mathrm{H} 1 \mathrm{D}$ & 109.5 \\
\hline $\mathrm{C} 4-\mathrm{C} 3-\mathrm{H} 3 \mathrm{~A}$ & 108.1 & $\mathrm{H} 1 \mathrm{C}-\mathrm{N} 1-\mathrm{H} 1 \mathrm{D}$ & 109.5 \\
\hline $\mathrm{O} 4-\mathrm{C} 4-\mathrm{C} 3$ & $111.44(12)$ & $\mathrm{C} 1-\mathrm{O} 1-\mathrm{C} 5$ & $114.09(11)$ \\
\hline $\mathrm{O} 4-\mathrm{C} 4-\mathrm{C} 5$ & $111.22(11)$ & $\mathrm{C} 1-\mathrm{O} 2-\mathrm{H} 2$ & 109.8 \\
\hline $\mathrm{C} 3-\mathrm{C} 4-\mathrm{C} 5$ & $109.58(12)$ & $\mathrm{C} 3-\mathrm{O} 3-\mathrm{H} 3$ & 100.2 \\
\hline $\mathrm{O} 4-\mathrm{C} 4-\mathrm{H} 4 \mathrm{~A}$ & 108.2 & $\mathrm{C} 4-\mathrm{O} 4-\mathrm{H} 4$ & 106.1 \\
\hline $\mathrm{C} 3-\mathrm{C} 4-\mathrm{H} 4 \mathrm{~A}$ & 108.2 & $\mathrm{C} 6-\mathrm{O} 5-\mathrm{H} 5$ & 111.2 \\
\hline $\mathrm{C} 5-\mathrm{C} 4-\mathrm{H} 4 \mathrm{~A}$ & 108.2 & & \\
\hline $\mathrm{O} 2-\mathrm{C} 1-\mathrm{C} 2-\mathrm{N} 1$ & $51.67(15)$ & $\mathrm{C} 2-\mathrm{C} 3-\mathrm{C} 4-\mathrm{C} 5$ & $53.63(15)$ \\
\hline $\mathrm{O} 1-\mathrm{C} 1-\mathrm{C} 2-\mathrm{N} 1$ & $174.97(12)$ & $\mathrm{O} 4-\mathrm{C} 4-\mathrm{C} 5-\mathrm{O} 1$ & $177.79(13)$ \\
\hline $\mathrm{O} 2-\mathrm{C} 1-\mathrm{C} 2-\mathrm{C} 3$ & $-70.19(16)$ & $\mathrm{C} 3-\mathrm{C} 4-\mathrm{C} 5-\mathrm{O} 1$ & $-58.55(16)$ \\
\hline $\mathrm{O} 1-\mathrm{C} 1-\mathrm{C} 2-\mathrm{C} 3$ & $53.11(18)$ & $\mathrm{O} 4-\mathrm{C} 4-\mathrm{C} 5-\mathrm{C} 6$ & $59.34(18)$ \\
\hline $\mathrm{N} 1-\mathrm{C} 2-\mathrm{C} 3-\mathrm{O} 3$ & $61.87(16)$ & $\mathrm{C} 3-\mathrm{C} 4-\mathrm{C} 5-\mathrm{C} 6$ & $-177.00(13)$ \\
\hline $\mathrm{C} 1-\mathrm{C} 2-\mathrm{C} 3-\mathrm{O} 3$ & $-176.18(13)$ & $\mathrm{O} 1-\mathrm{C} 5-\mathrm{C} 6-\mathrm{O} 5$ & $-57.79(16)$ \\
\hline $\mathrm{N} 1-\mathrm{C} 2-\mathrm{C} 3-\mathrm{C} 4$ & $-173.55(11)$ & $\mathrm{C} 4-\mathrm{C} 5-\mathrm{C} 6-\mathrm{O} 5$ & $62.12(17)$ \\
\hline $\mathrm{C} 1-\mathrm{C} 2-\mathrm{C} 3-\mathrm{C} 4$ & $-51.60(17)$ & $\mathrm{O} 2-\mathrm{C} 1-\mathrm{O} 1-\mathrm{C} 5$ & $60.77(16)$ \\
\hline $\mathrm{O} 3-\mathrm{C} 3-\mathrm{C} 4-\mathrm{O} 4$ & $-59.95(16)$ & $\mathrm{C} 2-\mathrm{C} 1-\mathrm{O} 1-\mathrm{C} 5$ & $-59.75(16)$ \\
\hline $\mathrm{C} 2-\mathrm{C} 3-\mathrm{C} 4-\mathrm{O} 4$ & $177.16(12)$ & $\mathrm{C} 6-\mathrm{C} 5-\mathrm{O} 1-\mathrm{C} 1$ & $-173.77(13)$ \\
\hline $\mathrm{O} 3-\mathrm{C} 3-\mathrm{C} 4-\mathrm{C} 5$ & $176.52(12)$ & $\mathrm{C} 4-\mathrm{C} 5-\mathrm{O} 1-\mathrm{C} 1$ & $63.51(16)$ \\
\hline
\end{tabular}

Hydrogen-bond geometry $(\AA, \circ)$

\begin{tabular}{|c|c|c|c|c|}
\hline$D-\mathrm{H} \cdots A$ & $D-\mathrm{H}$ & $\mathrm{H} \cdots A$ & $D \cdots A$ & $D-\mathrm{H} \cdots A$ \\
\hline $\mathrm{N} 1-\mathrm{H} 1 \mathrm{~B} \cdots \mathrm{O} 5^{\mathrm{i}}$ & 0.89 & 1.89 & $2.7772(17)$ & 172 \\
\hline $\mathrm{N} 1-\mathrm{H} 1 \mathrm{C} \cdots \mathrm{O} 4^{\mathrm{ii}}$ & 0.89 & 2.15 & $2.8930(19)$ & 141 \\
\hline $\mathrm{N} 1-\mathrm{H} 1 \mathrm{D} \cdots \mathrm{C} 11^{\mathrm{iii}}$ & 0.89 & 2.38 & $3.1744(13)$ & 149 \\
\hline $\mathrm{O} 2-\mathrm{H} 2 \cdots \mathrm{Cl} 1^{\mathrm{iv}}$ & 0.82 & 2.35 & $3.1448(12)$ & 162 \\
\hline $\mathrm{O} 3-\mathrm{H} 3 \cdots \mathrm{Cl} 1^{\mathrm{v}}$ & 0.84 & 2.35 & $3.1911(14)$ & 173 \\
\hline $\mathrm{O} 4-\mathrm{H} 4 \cdots \mathrm{Cl} 1$ & 0.82 & 2.35 & 3.1667 (14) & 175 \\
\hline $\mathrm{O} 5-\mathrm{H} 5 \cdots \mathrm{O} 3^{\mathrm{v}}$ & 0.81 & 1.95 & $2.7373(17)$ & 163 \\
\hline
\end{tabular}

Symmetry codes: (i) $-x, y+1 / 2,-z+2$; (ii) $-x+1, y+1 / 2,-z+2$; (iii) $x, y, z+1$; (iv) $x-1, y, z$; (v) $-x+1, y-1 / 2,-z+2$. 
supplementary materials

Fig. 1

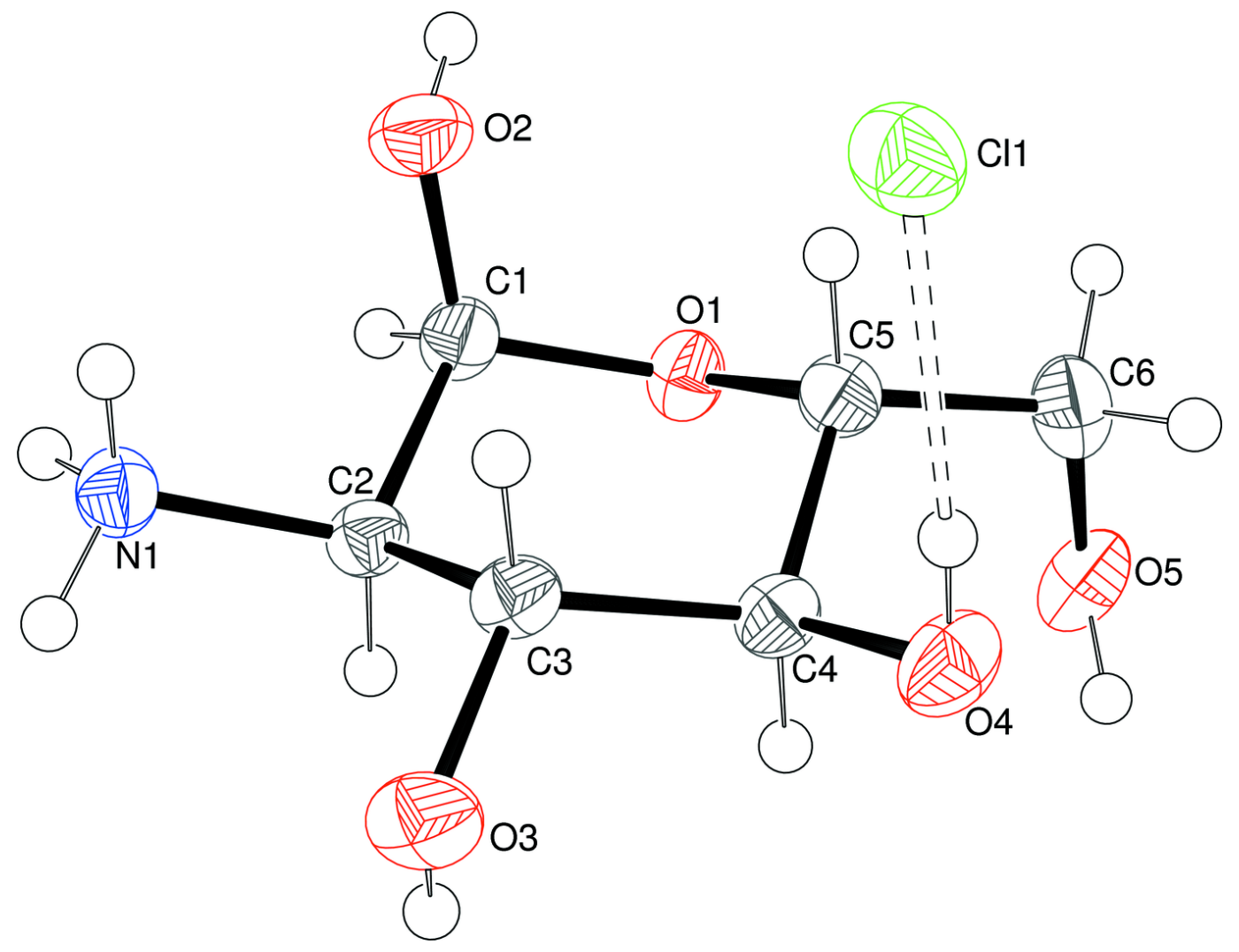

sup-6 
Fig. 2

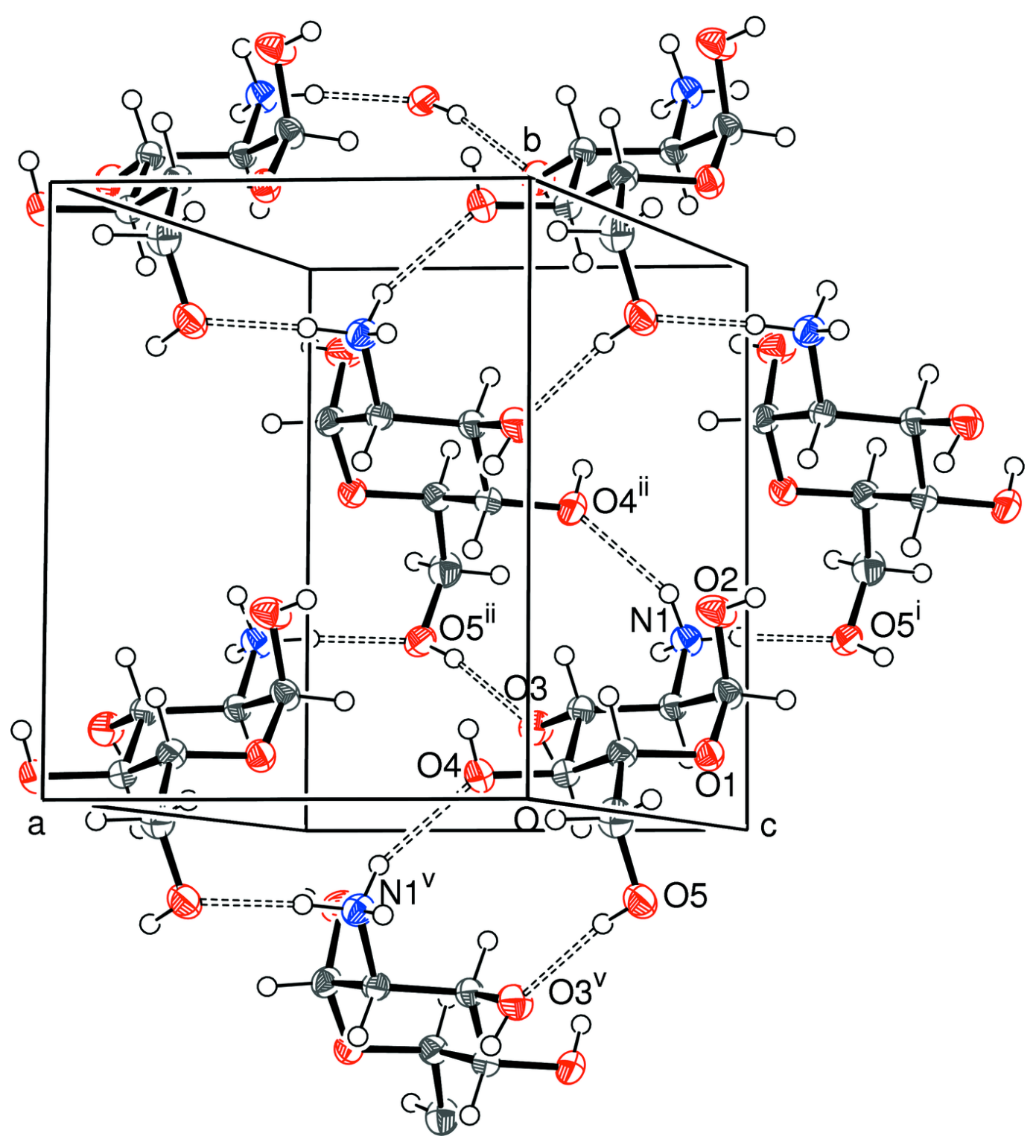

